\title{
Effect of Va Mycorrhizal Fungus and Bioformulations on Growth and Yield Attributes of Fennel under Northern Dry Zone (ZONE-3) of Karnataka
}

\author{
Shashidhar M. Dodamani ${ }^{1^{*}}$, N. K. Hegde ${ }^{1}$, Laxman Kukanoor ${ }^{2}$, \\ Chaya P. Patil ${ }^{1}$, T. B. Alloli ${ }^{1}$ and Krishna Kurubetta ${ }^{3}$ \\ ${ }^{1}$ Department of Horticulture, Kittur Rani Channamma College of Horticulture, Arabhavi, \\ University of Horticultural Sciences, Bagalkot, Karnataka, India \\ ${ }^{2}$ Kumbapur Farm Dharwad, UHS, Bagalkot, India \\ ${ }^{3}$ Agronomy, HRES, Devihosur, India \\ *Corresponding author
}

\section{A B S T R A C T}

\section{Keywords}

Fennel, VA

Mycorrhizal fungus, Bioformulations,

Northern dry zone,

Yield, Quality,

Economics

Article Info

Accepted:

07 November 2020

Available Online:

10 December 2020
Field trial on the effect of VAM and bioformulations on growth, yield and quality of fennel was carried out at KRCCH, Arabhavi, Karnataka during Rabi season of 2017 and 2018. There was significant variation was observed for growth and yield attributes of fennel due to application of VAM and bioformulations at 35, 70 days after sowing (DAS) and at harvest. The maximum plant height $(40.59,137.45$ and $171.47 \mathrm{~cm}$ respectively), number of branches (9.50 and 23.86 respectively). Maximum number of umbels per plant (18.06, 17.70 and 17.88), 1000 seed weight (7.96, 7.64 and $7.80 \mathrm{~g}$ ), seed yield $(12.50,12.00$ and $12.25 \mathrm{q} / \mathrm{ha}$ ), stover yield (19.30, 18.91 and $19.11 \mathrm{q} / \mathrm{ha})$ and biological yield (31.80, 30.91 and $31.36 \mathrm{q} / \mathrm{ha})$ were recorded by the treatment $\mathrm{T}_{9}$ (VAM $25 \mathrm{~kg} \mathrm{ha}^{-1}+$ Panchagavya $3 \%+$ Amrutpani 3\% + Vermiwash 10\% (Drench) + Mulch (Sugar cane trash) in 2017, 2018 and pooled data. While the minimum value for similar attributes were recorded in treatment $\mathrm{T}_{2}\left(\mathrm{VAM} 25 \mathrm{~kg} \mathrm{ha}^{-1}\right)$.

\section{Introduction}

India is known as the land of spices from the times immemorial and has been the global leader in the production, consumption and export of spices. Fennel (Foeniculum vulgare Mill.) is one of the popular seed spice in India mainly grown in rabi season. It is locally known as saunf and belongs to the family Apiaceae (Umbelliferae). Fennel is native to Southern Europe and Mediterranean region. It is a hardy perennial, but is grown as annual or biennial. It is cultivated throughout the temperate and subtropical regions of the world for its aromatic seeds which are used for culinary purpose. Fennel seed is small, oblong or cylindrical, 6-8 mm. long straight or slightly curved yellowish brown. It possesses an agreeable, aromatic and sweet aroma due to higher content of volatile oil (0.7 to $1.2 \%)$. The volatile oil contents mainly anethole $(50.03 \%)$ and fenchone $(2.67 \%)$. It is 
widely used as flavouring agent in culinary preparations, confectioneries, cordials and liquors. Fennel oil is also used as important ingredient in several allopathic as well as ayurvedic medicines which are used in diseases viz., diabetes, bronchitis and chronic coughs, treatment of kidney stones and is considered to have diuretic and galactogogue properties. The use of bio-fertilizers and bioformulations play an important role as they help in availability and supply of plant nutrients thereby, providing a scope for reduction in use of costly chemical fertilizers which pollute soil in long term (Kale et al., $1991)^{6}$. It is reported that 10 to 20 per cent of crop yield can be increased with biofertilizer application alone (Brown, 1972) ${ }^{4}$. The increasing concern about the environment and socio-economic impact of chemical agriculture has led to seek alternative practices for agricultural sustainability and marketability by progressive farmers. To minimize the adverse effects of conventional agriculture (e.g. polluted water and soil by chemical fertilizers, entering pesticides to the food chain, compaction of the soil by heavy machinery etc.), different alternative concepts of production have been developed. Currently, low input cropping systems and innovation of resource management are of the most important objectives of sustainable agriculture. Chemical free traditional farming technologies viz., bio-fertilizers, biodynamics, agnihotra (homa farming), panchagavya, Amrit Pani, rishi krishi, jeevamrutha etc., are gaining a new momentum not only in India, but also world over (Singh et al., 2007) ${ }^{10}$. These systems offer a means to address selfreliance, rural upliftment and conservation of natural resources. The connections between fungi and the roots of higher plants are referred to as mycorrhiza. Such interactions are mutualistic relationships undertaken by more than 80 per cent of plant species and approximately 6000 species of fungi. Arbuscular mycorrhizal (AM) fungi enable the host plant to establish and grow more efficiently under biotic and abiotic stress conditions, including drought through a series of complex communications between the host and fungus (Salam et al., 2017) ${ }^{9}$. Keeping this in view the present investigation was undertaken to study the Effect of VA Mycorrhizal fungus and bioformulations on growth and yield attributes of fennel under northern dry zone (Zone-3) of Karnataka.

\section{Materials and Methods}

The experiment was laid out in sandy loam soil with $\mathrm{pH}$ 6.5-8. Arabhavi is considered to have the benefit of both South-West and North-East monsoon. The mean annual rainfall for the past 20 years of this area is $449.25 \mathrm{~mm}$, distributed over a period of six to seven months from April to November. The monthly mean maximum temperature goes up to $38.45^{\circ} \mathrm{C}$ (March) and monthly mean minimum temperature drops down to $11.55^{\circ} \mathrm{C}$ in January. The meteorological data for the period of experimentation (Rabi 2017 and 2018) was recorded at the meteorological observatory of the Agricultural Research Station, Arabhavi (UAS, Dharwad) which is situated at $2 \mathrm{~km}$ from the college campus. The experiment was laid out in RCB Design replicated three times using Ajmeer Fennel-1 variety with nine treatments viz., $\mathrm{T}_{1}-90: 40$ : $30 \mathrm{~N}: \mathrm{P}: \mathrm{K} \mathrm{kg} \mathrm{ha}^{-1}$ (Check), $\mathrm{T}_{2}-$ VAM $25 \mathrm{~kg}$ $\mathrm{ha}^{-1}, \mathrm{~T}_{3}-$ 90: 40: $30 \mathrm{~N}: \mathrm{P}: \mathrm{K} \mathrm{kg} \mathrm{ha}^{-1}+\mathrm{VAM}$ $25 \mathrm{~kg} \mathrm{ha}{ }^{-1}, \mathrm{~T}_{4}-$ VAM $25 \mathrm{~kg} \mathrm{ha}^{-1}+$ Panchagavya 3\% (Drench), $\mathrm{T}_{5}-$ VAM $25 \mathrm{~kg}$ $\mathrm{ha}^{-1}+$ Amrutpani 3\% (Drench), $\mathrm{T}_{6}-$ VAM 25 $\mathrm{kg} \mathrm{ha}^{-1}+$ Panchagavya 3\% + Amrutpani 3\% (Drench), T 7 -90: 40: $30 \mathrm{~N}$ : P: $\mathrm{K} \mathrm{kg} \mathrm{ha}^{-1}+$ Vermiwash $10 \%$ (Drench), $\mathrm{T}_{8}-$ VAM $25 \mathrm{~kg}$ $\mathrm{ha}^{-1}+$ Vermiwash $10 \%$ (Drench), $\mathrm{T}_{9}-\mathrm{VAM}$ $25 \mathrm{~kg} \mathrm{ha}^{-1}+$ Panchagavya 3\% + Amrutpani $3 \%+$ Vermiwash 10\% (Drench) + Mulch (Sugar cane trash). A spacing of $50 \mathrm{~cm}$ between rows and $25 \mathrm{~cm}$ between the plants was followed. Glomus fasciculatum, a VA 
Mycorrhizal fungus was applied in the rows of nursery beds prior to sowing of seeds at one $\mathrm{kg} / \mathrm{plot}$. Five representative plants were selected at random from each plot for recording the observations at 35, 70 DAS and at harvest.

The average from these five plants was worked out for the statistical computation. The data recorded for various observations was subjected to statistical analysis using the Fischer's method of analysis of variance with 5 per cent level of significance for ' $F$ ' and ' $t$ ' tests. Pooled analysis of 2017 and 2018 was also carried out to draw conclusion. Wherever the ' $F$ ' test was significant, the critical difference (C.D.) values were worked out at 5 per cent level of significance (Panse and Sukhatme, 1985) ${ }^{7}$.

\section{Results and Discussion}

\section{Growth parameters}

Significant difference was observed for growth attributes due to application of VAM and bioformulations at 35, 70 days after sowing (DAS) and at harvest. The maximum plant height $(40.59,138.85$ and $171.47 \mathrm{~cm}$ respectively), number of branches (9.50 and 23.86 respectively), fresh weight (127.86, 417.43 and $907.83 \mathrm{~g}$ respectively), dry weight (27.87, 120.04 and 233.64 g respectively) and maximum days to 50 per cent flowering (94.00, 94.00 and 94.00 days) were recorded by the treatment $\mathrm{T}_{9}$ (VAM $25 \mathrm{~kg} \mathrm{ha}^{-1}+$ Panchagavya 3\%+ Amrutpani 3\% + Vermiwash 10\% (Drench) + Mulch (Sugar cane trash) in pooled data (Table 1-5).

This may be due to the fact that VAM and bioformulations have the ability to produce growth promoting substances and change in the metabolic activities which might have led to enhanced cell division and cell elongation leading to increased uptake of water and nutrients further resulting in maximum plant height, number of branches per plant, fresh weight and dry weight. In addition to the VAM and bioformulations, application of FYM which provides valuable organic nitrogen in addition to their beneficial influence in supplying the macro and micro nutrients, growth promoting substances, vitamins and improving physico-chemical properties of soil, which might have resulted in higher growth parameters in the present study.

Similar results were also reported by Biradar $(2003)^{2}$, Rajamani et al., $(2007)^{8}$, Velmurugan and Chezhiyan (2008) ${ }^{11}$ and Duragannavar $(2005)^{5}$ who reported that plants which received panchagavya (soil and foliar application) and Amrit pani (soil application) produced significantly higher plant height $(146.69 \mathrm{~cm})$, stem girth $(28.94 \mathrm{~cm})$ and number of leaves (52.33) in papaya cv. Red Lady.

\section{Yield and yield attributes}

Significant difference was observed for yield and yield attributes due to VAM and bioformulations viz., maximum number of umbels per plant (18.06, 17.70 and 17.88), number of umbellets per plant (236.40, 231.67 and 234.04), number of seeds per umbel (821.00, 804.58 and 812.79), 1000 seed weight $(7.96,7.64$ and $7.80 \mathrm{~g})$, seed yield per plant $(38.10,37.34$ and $37.72 \mathrm{~g})$, seed yield (12.50, 12.00 and $12.25 \mathrm{q} / \mathrm{ha})$, stover yield (19.30, 18.91 and $19.11 \mathrm{q} / \mathrm{ha})$ and biological yield (31.80, 30.91 and $31.36 \mathrm{q} / \mathrm{ha})$ were recorded by the treatment $\mathrm{T}_{9}$ (VAM 25 $\mathrm{kg} \mathrm{ha}^{-1}+$ Panchagavya 3\% + Amrutpani 3\% + Vermiwash 10\% (Drench) + Mulch (Sugar cane trash) and there was no significant difference observed among the treatments for harvest index in 2017, 2018 and pooled data respectively (Table 6-8). 
Table.1 Plant height in fennel at different growth stages as influenced by the application of VAM and bioformulations during rabi 2017 and 2018

\begin{tabular}{|c|c|c|c|c|c|c|c|c|c|}
\hline \multirow[t]{3}{*}{ Treatments } & \multicolumn{9}{|c|}{ Plant height (cm) } \\
\hline & \multicolumn{3}{|c|}{35 DAS } & \multicolumn{3}{|c|}{70 DAS } & \multicolumn{3}{|c|}{ At Harvest } \\
\hline & 2017 & 2018 & Pooled & 2017 & 2018 & Pooled & 2017 & 2018 & Pooled \\
\hline $\begin{array}{c}\mathrm{T}_{1-9} 90: 40: 30 \mathrm{~N}: \text { P: K kg ha } \\
{ }^{-} \text {(Check) }\end{array}$ & 33.59 & 32.92 & 33.25 & 120.50 & 118.09 & 119.30 & 148.81 & 145.84 & 147.33 \\
\hline T $_{2}-$ VAM $25 \mathrm{~kg} \mathrm{ha}^{-1}$ & 32.44 & 31.79 & 32.11 & 115.01 & 112.70 & 113.85 & 142.02 & 139.18 & 140.60 \\
\hline 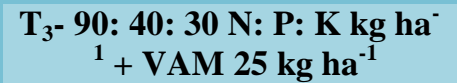 & 38.31 & 37.55 & 37.93 & 131.06 & 128.44 & 129.75 & 161.86 & 158.62 & 160.24 \\
\hline 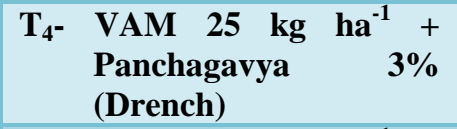 & 33.21 & 32.55 & 32.88 & 115.97 & 113.65 & 114.81 & 143.41 & 140.54 & 141.98 \\
\hline $\begin{array}{llr}\text { T }_{5^{-}} & \begin{array}{l}\text { VAM 25 kg } \\
\text { Amrutpani } \\
\text { (Drench) }\end{array} & \text { ha }^{-1}+ \\
& & \end{array}$ & 36.79 & 36.05 & 36.42 & 125.83 & 123.32 & 124.57 & 155.40 & 152.29 & 153.84 \\
\hline $\begin{array}{llr}\text { T}_{6-} & \begin{array}{l}\text { VAM } \\
\text { Panchagavya }\end{array} & \text { ha }^{-1}+ \\
& \mathbf{3 \%}+ \\
\text { Amrutpani } & + \\
\text { (Drench) } & & 3 \%\end{array}$ & 39.77 & 38.97 & 39.37 & 136.04 & 133.32 & 134.68 & 168.00 & 164.64 & 166.32 \\
\hline $\begin{aligned} \text { T}_{7}-90: & \text { 40: } 30 \mathrm{~N}: \text { P: } \mathrm{K} \mathrm{kg} \mathrm{ha} \\
& + \text { Vermiwash } 10 \% \\
& + \text { (Drench) }\end{aligned}$ & 35.17 & 34.47 & 34.82 & 123.13 & 120.66 & 121.89 & 152.05 & 149.01 & 150.53 \\
\hline 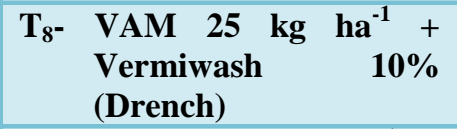 & 32.87 & 32.21 & 32.54 & 118.44 & 116.07 & 117.26 & 146.27 & 143.34 & 144.80 \\
\hline $\begin{array}{lll}\text { T}_{9^{-}} & \begin{array}{l}\text { VAM } \\
\text { Panchagavya }\end{array} \text { ha }^{-1}+ \\
& \mathbf{3 \%}+ \\
\text { Amrutpani } 3 \% & + \\
\text { Vermiwash } & \mathbf{1 0 \%} \\
\text { (Drench) + Mulch } & \end{array}$ & 41.00 & 40.18 & 40.59 & 140.25 & 137.45 & 138.85 & 173.20 & 169.74 & 171.47 \\
\hline S.Em. \pm & 1.21 & 1.07 & 1.14 & 1.83 & 3.91 & 1.94 & 1.99 & 3.19 & 1.63 \\
\hline CD at $5 \%$ & 3.63 & 3.19 & 3.41 & 5.47 & 11.72 & 5.81 & 5.95 & 9.57 & 4.90 \\
\hline CV (\%) & 13.95 & 13.68 & 13.81 & 12.22 & 12.80 & 12.24 & 12.80 & 11.95 & 12.23 \\
\hline
\end{tabular}

Note: 1. Bioformulations viz., Panchagavya, amrutpani and vermiwash were drenched at monthly interval from the day of sowing up to $2^{\text {nd }}$ harvest

2. Common dose of FYM (12.5 $\left.\mathrm{tha}^{-1}\right)$ was applied at the time of sowing 
Table.2 Number of branches in fennel at different growth stages as influenced VAM and bioformulations during rabi 2017 and 2018

\begin{tabular}{|c|c|c|c|c|c|c|}
\hline \multirow[t]{3}{*}{ Treatments } & \multicolumn{6}{|c|}{ Number of branches/plant } \\
\hline & \multicolumn{3}{|c|}{70 DAS } & \multicolumn{3}{|c|}{ At Harvest } \\
\hline & 2017 & 2018 & Pooled & 2017 & 2018 & Pooled \\
\hline $\mathrm{T}_{1}$ - 90: 40: $30 \mathrm{~N}: \mathrm{P}: \mathrm{K} \mathrm{kg} \mathrm{ha}^{-1}$ (Check) & 8.25 & 8.08 & 8.17 & 20.71 & 20.29 & 20.50 \\
\hline T $_{2^{-}}$VAM $25 \mathrm{~kg} \mathrm{ha}^{-1}$ & 7.75 & 7.60 & 7.68 & 19.31 & 18.92 & 19.12 \\
\hline $\mathrm{T}_{3^{-}}$90: 40: $30 \mathrm{~N}: \mathrm{P}: \mathrm{K} \mathrm{kg} \mathrm{ha}^{-1}+\mathrm{VAM} 25 \mathrm{~kg} \mathrm{ha}^{-1}$ & 8.97 & 8.79 & 8.88 & 22.52 & 22.07 & 22.30 \\
\hline T$_{4}-$ VAM $25 \mathrm{~kg} \mathrm{ha}^{-1}+$ Panchagavya $3 \%$ (Drench) & 7.87 & 7.72 & 7.79 & 19.76 & 19.37 & 19.57 \\
\hline T $_{5}$ VAM $25 \mathrm{~kg} \mathrm{ha}^{-1}+$ Amrutpani 3\% (Drench) & 8.61 & 8.44 & 8.53 & 21.62 & 21.19 & 21.41 \\
\hline $\begin{array}{l}\text { T6- }_{6} \text { VAM } 25 \mathrm{~kg} \mathrm{ha}^{-1}+\text { Panchagavya 3\% + Amrutpani 3\% } \\
\text { (Drench) }\end{array}$ & 9.31 & 9.13 & 9.22 & 23.38 & 22.91 & 23.14 \\
\hline $\mathrm{T}_{7}$ 90: 40: $30 \mathrm{~N}: \mathrm{P}: \mathrm{K} \mathrm{kg} \mathrm{ha}^{-1}+$ Vermiwash 10\% (Drench) & 8.43 & 8.26 & 8.34 & 21.16 & 20.73 & 20.95 \\
\hline T $_{8}$ VAM $25 \mathrm{~kg} \mathrm{ha}^{-1}+$ Vermiwash $10 \%$ (Drench) & 7.82 & 7.66 & 7.74 & 19.63 & 19.24 & 19.43 \\
\hline 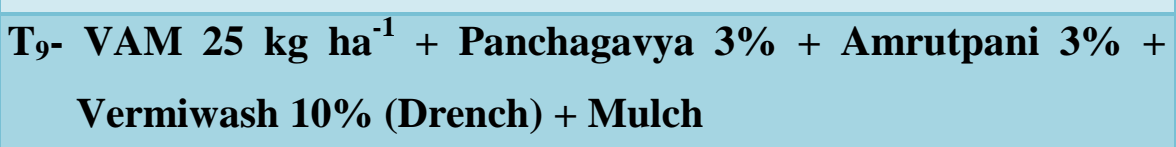 & 9.60 & 9.41 & 9.50 & 24.10 & 23.62 & 23.86 \\
\hline S.Em. \pm & 0.22 & 0.17 & 0.12 & 0.44 & 0.42 & 0.25 \\
\hline CD at $5 \%$ & 0.66 & 0.50 & 0.36 & 1.33 & 1.25 & 0.76 \\
\hline CV (\%) & 9.96 & 12.31 & 10.74 & 13.76 & 12.38 & 12.87 \\
\hline
\end{tabular}

Note: 1 . Bioformulations viz., Panchagavya, amrutpani and vermiwash were drenched at monthly interval from the day of sowing up to $2^{\text {nd }}$ harvest

2. Common dose of FYM $\left(12.5 \mathrm{tha}^{-1}\right)$ was applied at the time of sowing 
Table.3 Fresh weight of fennel plant at different growth stages as influenced VAM and bioformulations during rabi 2017 and 2018

\begin{tabular}{|c|c|c|c|c|c|c|c|c|c|}
\hline \multirow[t]{3}{*}{ Treatments } & \multicolumn{9}{|c|}{ Fresh weight $(\mathrm{g}) / \mathrm{plant}$} \\
\hline & \multicolumn{3}{|c|}{35 DAS } & \multicolumn{3}{|c|}{70 DAS } & \multicolumn{3}{|c|}{ At Harvest } \\
\hline & 2017 & 2018 & Pooled & 2017 & 2018 & Pooled & 2017 & 2018 & Pooled \\
\hline $\begin{array}{l}\mathrm{T}_{1-} \text { 90: 40: } 30 \mathrm{~N}: \mathrm{P}: \mathrm{K} \mathrm{kg} \mathrm{ha}^{-1} \\
\text { (Check) }\end{array}$ & 110.97 & 108.75 & 109.86 & 362.28 & 355.04 & 358.66 & 787.89 & 772.13 & 780.01 \\
\hline $\mathrm{T}_{2^{-}}$VAM $25 \mathrm{~kg} \mathrm{ha}^{-1}$ & 103.47 & 101.41 & 102.44 & 337.83 & 331.07 & 334.45 & 734.70 & 720.01 & 727.35 \\
\hline $\begin{array}{l}\text { T}_{3^{-}} \text {90: 40: } 30 \mathrm{~N}: \text { P: } \mathrm{K} \mathrm{kg} \mathrm{ha}^{-1}+ \\
\text { VAM } 25 \mathrm{~kg} \mathrm{ha}^{-1}\end{array}$ & 120.69 & 118.28 & 119.48 & 394.03 & 386.15 & 390.09 & 856.94 & 839.80 & 848.37 \\
\hline $\mathrm{T}_{4^{-}} \underset{\text { Panchagavya }}{\mathrm{VAM}} \underset{3 \%}{25}$ (Drench) $^{\mathrm{ha}^{-1}}+$ & 105.92 & 103.80 & 104.86 & 345.80 & 338.88 & 342.34 & 752.03 & 736.99 & 744.51 \\
\hline $\begin{array}{l}\text { T}_{5-} \text { VAM } 25 \mathrm{~kg} \mathrm{ha}^{-1}+\text { Amrutpani } \\
\text { 3\% (Drench) }\end{array}$ & 115.87 & 113.56 & 114.71 & 378.30 & 370.74 & 374.52 & 822.73 & 806.28 & 814.51 \\
\hline $\begin{array}{l}\text { T }_{6^{-}} \underset{\text { PaMchagavya }}{\text { VAM }} \quad 25 \quad \text { kg } \\
\text { 3\% } \\
\text { 3\% (Drench) }\end{array}$ & 125.28 & 122.77 & 124.02 & 409.00 & 400.82 & 404.91 & 889.49 & 871.70 & 880.60 \\
\hline $\begin{array}{l}\text { T}_{7} \text { 90: } 40: 30 \text { N: P: } \mathrm{K} \mathrm{kg} \mathrm{ha}^{-1}+ \\
\text { Vermiwash 10\% (Drench) }\end{array}$ & 113.38 & 111.11 & 112.25 & 370.17 & 362.76 & 366.46 & 805.03 & 788.93 & 796.98 \\
\hline $\begin{array}{l}\text { T}_{8}-\text { VAM } 25 \mathrm{~kg} \mathrm{ha}^{-1}+\text { Vermiwash } \\
10 \% \text { (Drench) }\end{array}$ & 109.07 & 106.89 & 107.98 & 343.43 & 336.57 & 340.00 & 746.90 & 731.96 & 739.43 \\
\hline $\begin{array}{l}\text { T9- VAM } 25 \mathrm{~kg} \mathrm{ha}^{-1}+\text { Panchagavya } \\
\text { 3\% + Amrutpani 3\% + } \\
\text { Vermiwash } 10 \% \text { (Drench) }+ \\
\text { Mulch }\end{array}$ & 129.15 & 126.57 & 127.86 & 421.65 & 413.22 & 417.43 & 917.00 & 898.66 & 907.83 \\
\hline S.Em. \pm & 1.75 & 2.39 & 1.27 & 3.44 & 3.03 & 2.46 & 5.61 & 6.59 & 4.64 \\
\hline CD at $5 \%$ & 5.25 & 7.18 & 3.80 & 10.32 & 9.09 & 7.37 & 16.83 & 19.76 & 13.91 \\
\hline $\mathrm{CV}(\%)$ & 15.32 & 12.22 & 13.59 & 18.36 & 11.68 & 14.89 & 14.67 & 11.68 & 13.13 \\
\hline
\end{tabular}

Note: 1. Bioformulations viz., Panchagavya, amrutpani and vermiwash were drenched at monthly interval from the day of sowing up to $2^{\text {nd }}$ harvest

2. Common dose of FYM $\left(12.5 \mathrm{tha}^{-1}\right)$ was applied at the time of sowing 
Table.4 Dry weight of fennel plant at different growth stages as influenced VAM and bioformulations during rabi 2017 and 2018

\begin{tabular}{|c|c|c|c|c|c|c|c|c|c|}
\hline \multirow[t]{3}{*}{ Treatments } & \multicolumn{9}{|c|}{ Dry weight (g)/plant } \\
\hline & \multicolumn{3}{|c|}{35 DAS } & \multicolumn{3}{|c|}{70 DAS } & \multicolumn{3}{|c|}{ At Harvest } \\
\hline & 2017 & 2018 & Pooled & 2017 & 2018 & Pooled & 2017 & 2018 & Pooled \\
\hline $\begin{array}{l}\mathrm{T}_{1-} \text { 90: 40: } 30 \mathrm{~N}: \mathrm{P}: \mathrm{K} \mathrm{kg} \mathrm{ha} \mathrm{ha}^{-1} \\
\text { (Check) }\end{array}$ & 24.19 & 23.70 & 23.94 & 104.18 & 102.09 & 103.14 & 202.77 & 198.72 & 200.74 \\
\hline 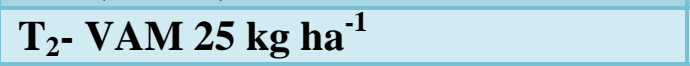 & 22.55 & 22.10 & 22.33 & 97.15 & 95.20 & 96.17 & 189.08 & 185.30 & 187.19 \\
\hline $\begin{array}{l}\text { T3- 90: 40: } 30 \mathrm{~N} \text { : P: } \mathrm{K} \mathrm{kg} \mathrm{ha}^{-1}+ \\
\text { VAM } 25 \mathrm{~kg} \mathrm{ha}^{-1}\end{array}$ & 26.31 & 25.78 & 26.04 & 113.31 & 111.04 & 112.18 & 220.54 & 216.13 & 218.34 \\
\hline $\begin{array}{l}\text { T}_{4} \text { - VAM } 25 \mathrm{~kg} \mathrm{ha}^{-1}+\text { Panchagavya } \\
\text { 3\% (Drench) }\end{array}$ & 23.09 & 22.62 & 22.85 & 99.44 & 97.45 & 98.44 & 193.54 & 189.67 & 191.61 \\
\hline $\begin{array}{l}\mathrm{T}_{5^{-}} \underset{\text { 3\% (Drench) }}{\text { VAM } 25 \mathrm{~kg} \mathrm{ha}^{-1}+\text { Amrutpani }} \\
\end{array}$ & 25.26 & 24.75 & 25.00 & 108.79 & 106.61 & 107.70 & 211.74 & 207.50 & 209.62 \\
\hline $\begin{array}{c}\text { T}_{6}-\text { VAM } 25 \mathrm{~kg} \mathrm{ha}^{-1}+\text { Panchagavya } \\
\text { 3\% + Amrutpani 3\% (Drench) }\end{array}$ & 27.31 & 26.76 & 27.03 & 117.61 & 115.26 & 116.44 & 228.92 & 224.34 & 226.63 \\
\hline $\begin{array}{l}\mathrm{T}_{7-} \text { 90: 40: } 30 \mathrm{~N}: \mathrm{P}: \mathrm{K} \mathrm{kg} \mathrm{ha} \mathrm{h}^{-1}+ \\
\text { Vermiwash 10\% (Drench) }\end{array}$ & 24.71 & 24.22 & 24.47 & 106.45 & 104.32 & 105.38 & 207.18 & 203.04 & 205.11 \\
\hline $\begin{array}{l}\text { T}_{8^{-}} \text {VAM } 25 \mathrm{~kg} \mathrm{ha}^{-1}+\text { Vermiwash } \\
10 \% \text { (Drench) }\end{array}$ & 22.93 & 22.47 & 22.70 & 98.76 & 96.78 & 97.77 & 192.22 & 188.38 & 190.30 \\
\hline T9- VAM $25 \mathrm{~kg} \mathrm{ha}^{-1}+$ Panchagavya & 28.15 & 27.59 & 27.87 & 121.25 & 118.83 & 120.04 & 236.00 & 231.28 & 233.64 \\
\hline $\begin{array}{l}3 \%+\text { Amrutpani } 3 \%+ \\
\text { Vermiwash } 10 \% \text { (Drench) }+ \\
\text { Mulch }\end{array}$ & & & & & & & & & \\
\hline S.Em. \pm & 0.728 & 0.845 & 0.500 & 2.653 & 3.454 & 3.049 & 7.074 & 6.760 & 6.913 \\
\hline CD at $5 \%$ & 2.18 & 2.53 & 1.50 & 7.95 & 10.36 & 9.14 & 21.21 & 20.27 & 20.73 \\
\hline CV (\%) & 11.84 & 10.73 & 10.74 & 12.87 & 10.58 & 11.61 & 10.67 & 13.37 & 11.90 \\
\hline
\end{tabular}

Note: 1. Bioformulations viz., Panchagavya, amrutpani and vermiwash were drenched at monthly interval from the day of sowing up to $2^{\text {nd }}$ harvest 2. Common dose of FYM (12.5 $\left.\mathrm{tha}^{-1}\right)$ was applied at the time of sowing 
Table.5 Days to 50 per cent flowering, number of umbels per plant and number of umbellets per plant as influenced by the application of VAM and bioformulations during rabi 2017 and 2018

\begin{tabular}{|c|c|c|c|c|c|c|c|c|c|}
\hline \multirow[t]{2}{*}{ Treatments } & \multicolumn{3}{|c|}{$\begin{array}{l}\text { Days to } 50 \text { per cent } \\
\text { flowering }\end{array}$} & \multicolumn{3}{|c|}{ Number of umbels per plant } & \multicolumn{3}{|c|}{$\begin{array}{c}\text { Number of umbellets per } \\
\text { plant }\end{array}$} \\
\hline & 2017 & 2018 & Pooled & 2017 & 2018 & Pooled & 2017 & 2018 & Pooled \\
\hline $\begin{array}{l}\text { T}_{1-} \text { 90: 40: } 30 \mathrm{~N}: \text { P: } \mathrm{K} \text { kg ha } \\
\text { (Check) }\end{array}$ & 93.00 & 92.00 & 92.50 & 15.52 & 15.21 & 15.36 & 203.11 & 199.05 & 201.08 \\
\hline $\mathrm{T}_{2}-$ VAM $25 \mathrm{~kg} \mathrm{ha}^{-1}$ & 92.00 & 90.00 & 91.00 & 14.47 & 14.18 & 14.32 & 189.40 & 185.62 & 187.51 \\
\hline $\begin{array}{l}\text { T}_{3^{-}} \text {90: 40: } 30 \mathrm{~N}: \text { P: } \mathrm{K} \mathrm{kg} \mathrm{ha-1}^{-1} \\
\text { VAM } 25 \mathrm{~kg} \mathrm{ha}^{-1}\end{array}$ & 94.00 & 94.00 & 94.00 & 16.88 & 16.54 & 16.71 & 220.92 & 216.50 & 218.71 \\
\hline T$_{4^{-}} \underset{\text { Panchagavya }}{\text { VAM }} \underset{3 \%}{25} \mathrm{~kg}_{(\text {Drench) }}^{\mathrm{ha}^{-1}}+$ & 94.00 & 93.00 & 93.50 & 14.81 & 14.51 & 14.66 & 193.87 & 189.99 & 191.93 \\
\hline $\begin{array}{l}\text { T}_{5}-\text { VAM } 25 \mathrm{~kg} \mathrm{ha}^{-1}+\text { Amrutpani } \\
3 \% \text { (Drench) }\end{array}$ & 93.00 & 92.00 & 92.50 & 16.20 & 15.88 & 16.04 & 212.10 & 207.86 & 209.98 \\
\hline $\begin{array}{cccc}\text { T }_{6-} & \begin{array}{l}\text { VAM } \\
\text { Panchagavya }\end{array} & \text { ha }^{-1} & + \\
\text { Amrutpani 3\% } & \text { (Drench) } & +\end{array}$ & 94.00 & 93.00 & 93.50 & 17.52 & 17.17 & 17.34 & 229.31 & 224.72 & 227.01 \\
\hline $\begin{array}{l}\mathrm{T}_{7}-\text { 90: } 40: 30 \mathrm{~N}: \mathrm{P}: \mathrm{K} \mathrm{kg} \mathrm{ha}^{-1}+ \\
\text { Vermiwash 10\% (Drench) }\end{array}$ & 93.00 & 94.00 & 93.50 & 15.85 & 15.54 & 15.70 & 207.54 & 203.38 & 205.46 \\
\hline 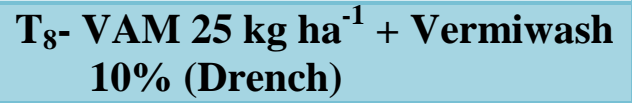 & 93.00 & 92.00 & 92.50 & 14.71 & 14.42 & 14.56 & 192.55 & 188.70 & 190.62 \\
\hline 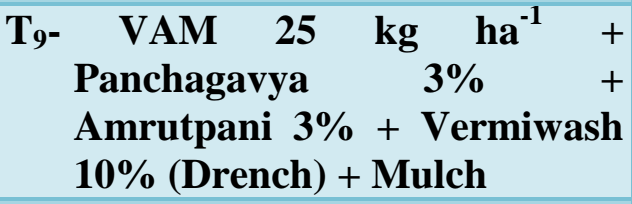 & 94.00 & 94.00 & 94.00 & 18.06 & 17.70 & 17.88 & 236.40 & 231.67 & 234.04 \\
\hline S.Em. \pm & 0.06 & 0.12 & 0.08 & 0.49 & 0.52 & 0.50 & 4.51 & 4.65 & 3.06 \\
\hline CD at $5 \%$ & 0.18 & 0.34 & 0.25 & 1.46 & 1.55 & 1.50 & 13.51 & 13.95 & 9.16 \\
\hline CV (\%) & 12.50 & 12.56 & 12.52 & 10.38 & 13.37 & 11.75 & 16.57 & 12.58 & 14.35 \\
\hline
\end{tabular}

Note: 1. Bioformulations viz., Panchagavya, amrutpani and vermiwash were drenched at monthly interval from the day of sowing up to $2^{\text {nd }}$ harvest 2. Common dose of FYM $\left(12.5 \mathrm{t} \mathrm{ha}^{-1}\right)$ was applied at the time of sowing 
Table.6 Number of umbels per plant and thousand seed weight in fennel at different growth stages as influenced by the application of VAM and bioformulations during rabi 2017 and 2018

\begin{tabular}{|c|c|c|c|c|c|c|}
\hline \multirow[t]{2}{*}{ Treatments } & \multicolumn{3}{|c|}{ Number of seeds/umbel } & \multicolumn{3}{|c|}{ Thousand seed weight (g) } \\
\hline & 2017 & 2018 & Pooled & 2017 & 2018 & Pooled \\
\hline $\mathrm{T}_{1}$ - 90: 40: $30 \mathrm{~N}: \mathrm{P}: \mathrm{K} \mathrm{kg} \mathrm{ha}^{-1}$ (Check) & 705.40 & 691.30 & 698.35 & 7.48 & 7.18 & 7.33 \\
\hline $\mathrm{T}_{2}-$ VAM $25 \mathrm{~kg} \mathrm{ha}^{-1}$ & 657.79 & 644.63 & 651.21 & 7.33 & 7.04 & 7.18 \\
\hline 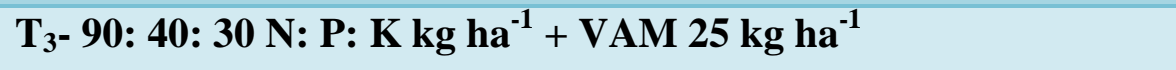 & 767.22 & 751.88 & 759.55 & 7.65 & 7.34 & 7.50 \\
\hline $\mathrm{T}_{4}-$ VAM $25 \mathrm{~kg} \mathrm{ha}^{-1}+$ Panchagavya $3 \%$ (Drench) & 673.30 & 659.84 & 666.57 & 7.39 & 7.09 & 7.24 \\
\hline T $^{-}$VAM $25 \mathrm{~kg} \mathrm{ha}^{-1}+$ Amrutpani 3\% (Drench) & 736.60 & 721.87 & 729.24 & 7.59 & 7.29 & 7.44 \\
\hline $\begin{array}{l}\text { T6- }_{\text {- }} \text { VAM } 25 \mathrm{~kg} \mathrm{ha}^{-1}+\text { Panchagavya 3\% + Amrutpani 3\% } \\
\text { (Drench) }\end{array}$ & 796.37 & 780.44 & 788.41 & 7.72 & 7.41 & 7.57 \\
\hline $\mathrm{T}_{7^{-}}$90: 40: $30 \mathrm{~N}: \mathrm{P}: \mathrm{K} \mathrm{kg} \mathrm{ha}^{-1}+$ Vermiwash 10\% (Drench) & 720.76 & 706.34 & 713.55 & 7.53 & 7.23 & 7.38 \\
\hline 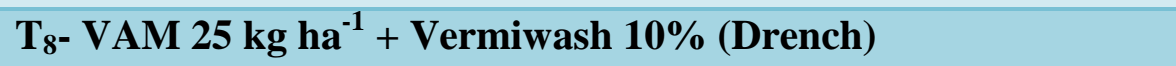 & 668.70 & 655.33 & 662.02 & 7.37 & 7.08 & 7.22 \\
\hline $\begin{array}{l}\text { T9- }_{9} \text { VAM } 25 \mathrm{~kg} \mathrm{ha}^{-1}+\text { Panchagavya } 3 \%+\text { Amrutpani } 3 \%+ \\
\text { Vermiwash } 10 \% \text { (Drench) + Mulch }\end{array}$ & 821.00 & 804.58 & 812.79 & 7.96 & 7.64 & 7.80 \\
\hline S.Em. \pm & 14.93 & 14.94 & 10.07 & 0.07 & 0.08 & 0.05 \\
\hline CD at $5 \%$ & 44.76 & 44.79 & 30.19 & 0.20 & 0.23 & 0.13 \\
\hline CV (\%) & 16.47 & 12.46 & 14.27 & 9.21 & 9.33 & 9.21 \\
\hline
\end{tabular}

Note: 1. Bioformulations viz., Panchagavya, amrutpani and vermiwash were drenched at monthly interval from the day of sowing up to $2^{\text {nd }}$ harvest

2. Common dose of FYM $\left(12.5 \mathrm{tha}^{-1}\right)$ was applied at the time of sowing 
Table.7 Seed yield per plant, seed and stover yield in fennel as influenced by the application of VAM and bioformulations during rabi 2017 and 2018

\begin{tabular}{|c|c|c|c|c|c|c|c|c|c|}
\hline \multirow[t]{2}{*}{ Treatments } & \multicolumn{3}{|c|}{ Seed yield/plant (g) } & \multicolumn{3}{|c|}{ Seed yield (q/ha) } & \multicolumn{3}{|c|}{ Stover yield (q/ha) } \\
\hline & 2017 & 2018 & Pooled & 2017 & 2018 & Pooled & 2017 & 2018 & Pooled \\
\hline $\begin{array}{l}\mathrm{T}_{1-} \text { 90: } 40: 30 \mathrm{~N}: \mathrm{P}: \mathrm{K} \mathrm{kg} \mathrm{ha}^{-1} \\
\text { (Check) }\end{array}$ & 32.74 & 32.08 & 32.41 & 10.74 & 10.31 & 10.53 & 18.16 & 17.80 & 17.98 \\
\hline T $_{2}-$ VAM $25 \mathrm{~kg} \mathrm{ha}^{-1}$ & 28.58 & 28.00 & 28.29 & 10.02 & 9.61 & 9.81 & 18.28 & 17.91 & 18.10 \\
\hline $\begin{array}{l}\text { T}_{3^{-}} \text {90: } 40: 30 \mathrm{~N}: \text { P: } \mathrm{K} \mathrm{kg} \mathrm{ha}^{-1}+ \\
\text { VAM } 25 \mathrm{~kg} \mathrm{ha}^{-1}\end{array}$ & 35.60 & 34.89 & 35.25 & 11.68 & 11.21 & 11.45 & 18.98 & 18.61 & 18.80 \\
\hline T $_{4^{-}} \underset{\text { Panchagavya }}{\text { VA\% }} \underset{\text { (Drench) }}{25} \mathrm{~kg}_{\text {ha }^{-1}}+$ & 31.25 & 30.62 & 30.93 & 10.25 & 9.84 & 10.05 & 18.40 & 18.03 & 18.22 \\
\hline T $_{5^{-}} \underset{\text { Amrutpani }}{\text { VA\% }} \underset{\text { (Drench) }}{25} \underset{\text { kg } \text { ha }^{-1}}{+}+$ & 34.18 & 33.50 & 33.84 & 11.22 & 10.77 & 10.99 & 18.72 & 18.35 & 18.54 \\
\hline 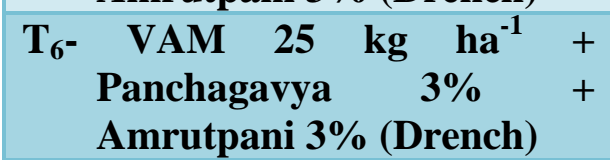 & 36.96 & 36.22 & 36.59 & 12.13 & 11.64 & 11.88 & 19.21 & 18.83 & 19.02 \\
\hline $\begin{array}{c}\text { T}_{7-90} \text { 90: 40: } 30 \mathrm{~N}: \mathrm{P}: \mathrm{K} \mathrm{kg} \mathrm{ha}^{-1}+ \\
\text { Vermiwash 10\% (Drench) }\end{array}$ & 33.45 & 32.78 & 33.11 & 10.97 & 10.53 & 10.75 & 17.94 & 17.58 & 17.76 \\
\hline T $_{8^{-}} \underset{\text { Vermiwash }}{\text { VAM }} \underset{10 \%}{25} \mathrm{~kg}_{\text {(Drench) }}^{\mathrm{ha}^{-1}}+$ & 31.03 & 30.41 & 30.72 & 10.18 & 9.77 & 9.98 & 18.29 & 17.92 & 18.11 \\
\hline $\begin{array}{c}\text { T9- }_{9}^{\text {VAM }} \text { 25 kg } \text { ha }^{-1}+ \\
\text { Panchagavya } \\
\text { Amrutpani 3\% + Vermiwash } \\
\text { 10\% (Drench) + Mulch }\end{array}$ & 38.10 & 37.34 & 37.72 & 12.50 & 12.00 & 12.25 & 19.30 & 18.91 & 19.11 \\
\hline 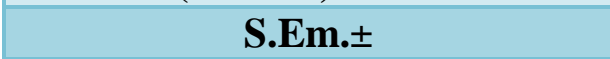 & 1.28 & 0.51 & 0.89 & 0.40 & 0.26 & 0.33 & 0.17 & 0.20 & 0.19 \\
\hline CD at $5 \%$ & 3.83 & 1.52 & 2.66 & 1.19 & 0.78 & 0.98 & 0.51 & 0.61 & 0.56 \\
\hline $\mathrm{CV}(\%)$ & 11.40 & 12.83 & 11.86 & 10.40 & 12.70 & 11.34 & 9.21 & 9.34 & 9.27 \\
\hline
\end{tabular}

Note: 1. Bioformulations viz., Panchagavya, amrutpani and vermiwash were drenched at monthly interval from the day of sowing up to $2^{\text {nd }}$ harvest

2. Common dose of FYM (12.5 tha $\left.\mathrm{ta}^{-1}\right)$ was applied at the time of sowing 
Table.8 Biological yield, harvest index and incidence of aphids in fennel seeds as influenced by the application of VAM and bioformulations during rabi 2017 and 2018

\begin{tabular}{|c|c|c|c|c|c|c|}
\hline \multirow[t]{2}{*}{ Treatments } & \multicolumn{3}{|c|}{ Biological yield (q/ha) } & \multicolumn{3}{|c|}{ Harvest index $(\%)$} \\
\hline & 2017 & 2018 & Pooled & 2017 & 2018 & Pooled \\
\hline $\mathrm{T}_{1}$ - 90: 40: $30 \mathrm{~N}: \mathrm{P}: \mathrm{K} \mathrm{kg} \mathrm{ha}^{-1}$ (Check) & 28.90 & 28.11 & 28.51 & 37.23 & 36.68 & 36.95 \\
\hline $\mathrm{T}_{2-}$ - VAM $25 \mathrm{~kg} \mathrm{ha}^{-1}$ & 28.29 & 27.53 & 27.91 & 35.10 & 34.66 & 34.88 \\
\hline $\mathrm{T}_{3^{-}}$90: 40: $30 \mathrm{~N}: \mathrm{P}: \mathrm{K} \mathrm{kg} \mathrm{ha}^{-1}+$ VAM $25 \mathrm{~kg} \mathrm{ha}^{-1}$ & 30.67 & 29.82 & 30.24 & 38.16 & 37.61 & 37.89 \\
\hline T $_{4}$ - VAM $25 \mathrm{~kg} \mathrm{ha}^{-1}+$ Panchagavya 3\% (Drench) & 28.65 & 27.87 & 28.26 & 35.85 & 35.31 & 35.58 \\
\hline 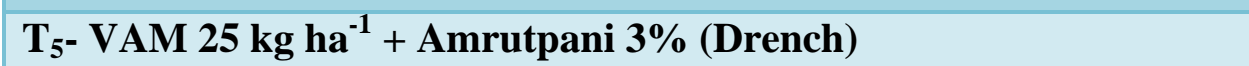 & 29.94 & 29.12 & 29.53 & 37.53 & 36.98 & 37.25 \\
\hline 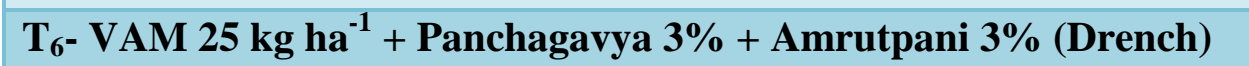 & 31.34 & 30.47 & 30.90 & 38.76 & 38.20 & 38.48 \\
\hline$T_{7-}$ 90: 40: $30 \mathrm{~N}: \mathrm{P}: \mathrm{K} \mathrm{kg} \mathrm{ha}^{-1}+$ Vermiwash 10\% (Drench) & 28.92 & 28.12 & 28.52 & 38.02 & 37.47 & 37.75 \\
\hline 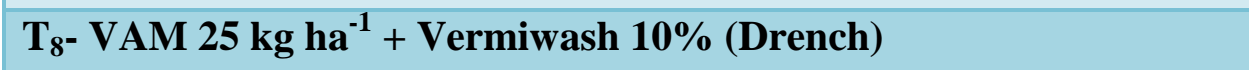 & 28.47 & 27.70 & 28.08 & 35.83 & 35.29 & 35.56 \\
\hline 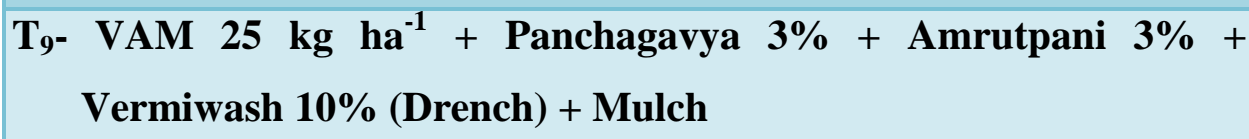 & 31.80 & 30.91 & 31.36 & 39.38 & 38.82 & 39.10 \\
\hline S.Em. \pm & 0.57 & 0.46 & 0.51 & 0.83 & 0.76 & 0.80 \\
\hline CD at $5 \%$ & 1.70 & 1.38 & 1.54 & NS & NS & NS \\
\hline CV (\%) & 8.93 & 10.30 & 9.58 & 10.61 & 10.46 & 10.53 \\
\hline
\end{tabular}

NS - Non-significant

Note: 1. Bioformulations viz., Panchagavya, amrutpani and vermiwash were drenched at monthly interval from the day of sowing up to $2^{\text {nd }}$ harvest

2. Common dose of FYM (12.5 $\left.\mathrm{tha}^{-1}\right)$ was applied at the time of sowing 
It is attributed to the effect of VAM in restrictive control on stomatal opening and closure, improvement of root water uptake characters and probably better nutrition status specially phosphorus and improved the ability of roots to absorb soil moisture, thus maintaining opened stomata in leaves and enhancing dry matter production. Enhanced water conductivity has been attributed to increased area for water uptake provided by hyphae in soil, due to VAM and biofertilizers symbiosis have led to higher yield (Bolandnazar and Hakiminia 2013) ${ }^{3}$. This conclusion corroborates the finding of Auge et al., (2004) ${ }^{1}$ in French bean. It is also reported that mycorrhizal plants under both well watered and deficit conditions had higher stomatal conductance and leaf growth rate compared to non-mycorrhizal ones. Mycorrhiza helps plants with such a shallow sparse root system to increase phosphorus uptake.

Significantly higher plant height (40.59, 138.85 and $171.47 \mathrm{~cm})$, fresh weight $(127.86$, 417.43 and $907.83 \mathrm{~g}$ ), dry weight (27.87, 120.04 and $233.64 \mathrm{~g}$ ) and maximum days to 50 per cent flowering (94.00 each) were recorded in the treatment $\mathrm{T}_{9}$ (VAM $25 \mathrm{~kg} \mathrm{ha}^{-1}$ + Panchagavya 3\% + Amrutpani 3\% + Vermiwash 10\% (Drench) + Mulch (Sugar cane trash) at 35, 70 days after sowing (DAS) and at harvest respectively in the pooled data.

Significantly higher number of umbels per plant (18.06, 17.70 and 17.88), number of umbellets per plant (236.40, 231.67 and 234.04), number of seeds per umbel (821.00, 804.58 and $812.79)$, thousand seed weight $(7.96,7.64$ and $7.80 \mathrm{~g})$, seed yield per plant $(38.10,37.34$ and $37.72 \mathrm{~g})$, seed yield per hectare $(12.50,12.00$ and $12.25 \mathrm{q} / \mathrm{ha}$ ) were recorded in the treatment $\mathrm{T}_{9}$ (VAM $25 \mathrm{~kg} \mathrm{ha}^{-1}+$ Panchagavya 3\% + Amrutpani 3\% + Vermiwash 10\% (Drench) + Mulch (Sugar cane trash) in 2017, 2018 and pooled data respectively
In conclusion the application of VAM and bio-formulations along with optimum dose of fertilizer influenced the growth, yield and yield attributes of fennel. Application of VAM (25 kg ha-1) along with foliar spray of Panchagavya (3\%), Amrutpani (3\%) + Vermiwash 10\% (Drench) + Mulch (Sugar cane trash) recorded the higher values for growth, yield and yield attributes of fennel

\section{References}

Auge, R. M., Sylvia, D. M., Park, S., Buttery, B. R., Saxton, A. M., Moore, J. L. and Cho, K. 2004. Partitioning mycorrhizal influence on water relations of Phaseolus vulgaris into soil and plant components. Canadian Journal of Botany. 82: 503-514.

Biradar, P. 2003. Effect of microbial consortia on growth and yield of banana ratoon crop, cv. Rajapuri. M. Sc. (Hort.) Thesis, Univ. Agric. Sci., Dharwad, Karnataka.

Bolandnazar, S. and Hakiminia, I. 2013. Impact of mycorrhizal fungi on $\mathrm{P}$ acquisition, yield and water use efficiency of onion under regulated deficit irrigation. Res. Plant Biol. 3(1): 18-23.

Brown, M. E. 1972. Plant growth substances produced by microorganisms of soil and rhizosphere. J. Appl. Bact. 35: 443.

Duragannavar, M. P. 2005. Effect of bioformulations on growth and yield of papaya cv. Red Lady. M. Sc. (Hort.) Thesis, Univ. Agric. Sci., Dharwad, Karnataka.

Kale, R. N., Bano, K. and Satyavati, G. P. 1991. Influence of vermicompost application on growth and yield of cereals, vegetables and ornamental plants. Final Report of KSCSI Project. 34B. 34-78.

Krishnamurthy, D. and Sharanappa. 2005. 
Effect of sole and integrated use of improved composts and NPK fertilizers on the quality, productivity and shelf life of Bangalore Rose Red onion (Alium cepa L.). Mysore J. Agril. Sci. 39(2): 355-361.

Meena, S. S., Mehta, R. S., Meena, R. D., Meena, N. K. and Singh, B. 2013. Effect of sowing time and crop geometry on growth and seed yield of dill (Anethum sowa L.) Int. J. Seed Spices. 3(2): 81-84.

Panse, U. G. and Sukhatme, B. V. 1985. Statistical methods for Agricultural workers. Indian Council of Agricultural Research, New Delhi. pp. 100-161.

Rajamani, K., Kamalkumar, R., Shoba, N. and Balakrishnamurthy, G. 2007. Influence of organic manure and bioregulates on growth and yield of turmeric var. BSR-2. South Indian Hort. 55 (1-6): 170-178.

Rana, S. C., Pandita, V. K. and Sanjai, S. 2015. Influence of spacing and number of leaf cutting on seed yield in fenugreek. Legume Res. 38(6): 858:860.

Salam, E. A., Alatar, A. and El-Sheikh, M. A. 2017. Inoculation with arbuscular mycorrhizal fungi alleviates harmful effects of drought stress on damask rose. Saudi J. Biol. Sci. 9(3): 44-49.

Singh, A. and Amin, A. U. 2015. Response of drilled rabi fennel (Foeniculum vulgare Mill) to spacing under varying levels of nitrogen. International J. Seed Spices. 5(1): 102-104.

Singh, B., Ranjan, S. and Ramchandra. 2007. Response of panchagavya and manchurian mushroom tea on floral characters in tuberose (Polyanthus tuberose Linn.) cv. Pearl Double. J. Ornamental Hort. 10 (4): 250-254.

Velmurugan, M.. and Chezhiyan, N. 2008. Effect of organic manures and biofertilizers on growth, yield and quality of turmeric (Curcuma longa L.) cv. BSR-2. South Indian Hort. 53(1-6): 392-396.

\section{How to cite this article:}

Shashidhar M. Dodamani, N. K. Hegde, Laxman Kukanoor, Chaya P. Patil, T. B. Alloli and Krishna Kurubetta. 2020. Effect of Va Mycorrhizal Fungus and Bioformulations on Growth and Yield Attributes of Fennel under Northern Dry Zone (ZONE-3) of Karnataka. Int.J.Curr.Microbiol.App.Sci. 9(12): 756-768. doi: https://doi.org/10.20546/ijcmas.2020.912.091 